\title{
Update zur personalisierten Diagnostik und Behandlung krebskranker Kinder
}

\author{
Update: Personalized Diagnostics and Therapies for Pediatric Cancer Patients
}

Notwendigkeit der Therapieoptimierung bei Krebserkrankungen im Kindes- und Jugendalter

$\nabla$

Die erfreuliche Entwicklung der langfristigen Heilungsraten der Krebserkrankungen im Kindes- und Jugendalter während der letzten Jahrzehnte auf heute $>80 \%$ gehört zu den Erfolgsgeschichten der modernen Medizin [5]. Der Preis des Therapieerfolges ist jedoch noch immer zu hoch: teilweise lebensbedrohliche Nebenwirkungen und gravierende Spätfolgen können mit der Behandlung einhergehen. Insbesondere die zahlreichen möglichen Spätfolgen nach Chemotherapie und Bestrahlung (Zweitmalignome, Nephrotoxizität, Kardiotoxizität, Hepatotoxizität, endokrinologische Störungen, Ototoxizität, Infertilität [20], Wachstumsstörungen, Entwicklungsverzögerungen, intellektuelle Defizite usw.) geben Anlass zur Sorge. Mit den Nebenwirkungen konventioneller Chemo- oder Strahlentherapie beschäftigen sich in dieser Ausgabe der Klinischen Pädiatrie 2 Beiträge (M. Götte et al., S. 133137 und C. Pfitzer et al., S. 138-144). Hinzu kommt, dass die Möglichkeiten der klassischen 3-Säulen Therapie (Chirurgie, Chemotherapie und Strahlentherapie) ausgeschöpft zu sein scheinen, da die Heilungsraten bei den meisten Krebserkrankungen des Kindes- und Jugendalters trotz Intensivierung der Therapie auf dem erreichten hohen Niveau entweder stagnieren oder sich nur sehr langsam weiter verbessern [5]. Inzwischen kann über Erfolge mit Medikamenten berichtet werden, die in die Signalwege von leukämischen Zellen bei Kindern und Jugendlichen zielgerichtet eingreifen und im Einzelfall z.B. nach schwerer Komplikation - besser verträglich sind als die aktuell empfohlene Chemotherapie (E. Müller et al. S. 172-173). Allerdings ist bei breiter Anwendung zielgerichteter Medikamente bei Kindern mit Nebenwirkungen zu rechnen, die bei gleicher Indikation bei Erwachsenen nicht zu erwarten sind (A. Ulmer et al. S. 120-126).

In den letzten Jahren ist das Wissen über die molekularen Ursachen der Entstehung und Progression der Krebserkrankungen sprunghaft gestiegen $[1,3,14,16,21,23,24,30,32,35-37,40,46]$. Durch den Einsatz moderner Hochdurchsatztechnologien, die heute eine parallele Untersuchung der Aktivität aller Gene und sogar epigenetischer Veränderungen eines Gewebes erlauben, konnten bereits in vielen Tumorarten fehlregulierte Komponenten bestimmter zellulärer Signalwege identifiziert und die Funktion entsprechender Gene oder Proteine charakterisiert werden $[1,3,14,16,21,23,24,30,32,35-37,40,46]$. Diese neuen Erkenntnisse ebnen den Weg für noch präzisere Diagnosen und individuelle, für jeden Patienten maßgeschneiderte Behandlungsansätze. Klinische Bedeutung für diese neuen Behandlungsansätze erfährt die Erforschung eines Signalwegs durch das Vorhandensein spezifischer Inhibitoren, die auf verschiedenen Stufen der Signalkaskade wirken und vielversprechende neue Medikamente darstellen können. Daher ist die gezielte Forschung auf dem Gebiet der pädiatrischen molekularen Onkologie im Rahmen der einheitlichen Therapieoptimierungsprotokolle und diagnosebezogenen klinischen Register als Grundlage personalisierter Diagnostik- und Behandlungskonzepte zwingend notwendig.

\section{Personalisierte Medizin in der Kinderonkologie \\ $\nabla$}

Bereits seit einiger Zeit haben Onkologen die Vision einer individualisierten Diagnostik und Therapie, die in den letzten Jahren auch unter den Begriffen personalisierte Medizin, Präzisionsmedizin oder Systemmedizin bekannt wurde. Personalisierte Medizin bedeutet kurz: die richtige Therapie für den richtigen Patienten zur richtigen Zeit. Voraussetzung dafür sind präzise und molekular basierte diagnostische Möglichkeiten, die eine gezielte statt breit gestreute Therapie ermöglichen. Hierunter versteht man beispielsweise eine Biomarker-gestützte Diagnostik, die hilft herauszufinden, ob ein Patient von einer bestimmten Therapie profitieren wird. Solche Marker befinden sich traditionell auf der Ebene einzelner Gene, charakteristischer Genmutationen oder der Proteine. Anstelle einzelner Biomarker finden sich allerdings mehr und mehr komplexe molekulare Signaturen auf der Genom- Transkriptom- Epigenom- oder Proteomebene, die auf der Basis komplizierter bioinformatischer Verfahren in Form sogenannter Klassifikatoren oder Prädiktoren erstellt wurden.

Mithilfe molekularer Klassifikatoren lassen sich bei vielen Tumorarten mit hoher Zuverlässigkeit neue Untergruppen definieren, die einen unterschiedlichen Bedarf an gezielten Therapieformen und auch häufig eine unterschiedliche Heilungsaussicht haben. Die Basis für die Klassifikatoren bilden effiziente neue Technologien, welche die gleichzeitige Untersuchung zehntausender Gene in einer kleinen Tumorprobe in einem einzigen Experiment mithilfe von Microarrays oder genomweiter Sequenzierungen der DNA ermöglichen. Die neuen Methoden des „Next Generation 
Sequencing“ erlauben in Zukunft eine noch feinere und detailliertere Darstellung der genetischen Auffälligkeiten eines Tumors. Als herausragendes Beispiel aus der Kinderonkologie für die Nutzung dieser neuen molekularen Diagnostik-Instrumente darf dabei die Erstellung einer neuen molekularen Klassifikation der Hirntumoren gelten [18, 19,25, 26,33, 34,39,42,44]. Für die bahnbrechenden Arbeiten seiner Arbeitsgruppe, die mit hoher internationaler Sichtbarkeit wesentlich zu zahlreichen neuen molekularen Erkenntnissen im Gebiet pädiatrischer Hirntumoren beigetragen haben, wurde unserem Kollegen Prof. Dr. med. Stefan Pfister, DKFZ Heidelberg, in diesem Jahr sehr verdient der Translationale Krebspreis der Deutschen Krebsgesellschaft verliehen, zu dem wir an dieser Stelle noch einmal sehr herzlich gratulieren!

Molekulare Prädiktoren stützen sich heute ebenfalls größtenteils auf den Nachweis genomischer oder transkriptomischer Veränderungen in den Tumorzellen und eignen sich für die zuverlässige Vorhersage des Therapieansprechens individueller Tumoren. Auf diese Weise können sie für die frühe Identifizierung von Patienten genutzt werden, für die einerseits eine weniger aggressive Therapie ausreicht oder die andererseits von einer aggressiveren oder experimentellen Therapieformen profitieren könnten. Bereits gelungen ist die verbesserte Risikostratifizierung von Patienten auf der Basis molekularer Prädiktoren auf Genom-, Transkriptom- und Epigenomebene beim Neuroblastom $[6,7,9,10,17,27,28,38,43]$. Durch die Untersuchung umfassender Tumorkollektive in Deutschland, Europa, den USA und Japan mithilfe von Microarrays und ArrayCGH-Analysen sind genetische Signaturen identifiziert worden, die die Einschätzung des Rückfallrisikos bei einem Neuroblastom deutlich verbessern $[6,7,9,10,17,27,28,38,43]$. So wird es zukünftig möglich sein, sowohl die Gruppe der Beobachtungspatienten als auch die Gruppe der Hochrisikopatienten mit hoher Wahrscheinlichkeit für ein Therapieversagen bereits zum Zeitpunkt der Diagnosestellung verlässlich zu definieren. In Deutschland sind hierzu durch die konsekutiv aufeinander aufbauenden Therapieoptimierungsstudien für die Diagnostik und Behandlung des Neuroblastoms und das flächendeckende Neuroblastom-Screening wesentliche Voraussetzungen geschaffen worden, die über Jahrzehnte durch die Deutsche Krebshilfe gefördert worden sind. Die Untersuchung der Tumoren dieser genau definierten und gut dokumentierten Patienten im Nationalen Genomforschungsnetz wird durch die langjährige Förderung eines multizentrischen Neuroblastom-Forschungsverbunds seitens des BMBF ermöglicht. Es besteht die realistische Hoffnung, dass die weitere Nutzung der neuen Sequenzierungstechnologien und weiterer Analysen [2] dazu führen wird, für solide Tumoren eine ähnlich präzise MRD-Diagnostik zu etablieren, wie es für die akute lymphoblastische Leukämie schon vor Jahren gelungen ist.

Moderne Therapeutika in der personalisierten Therapie richten sich auf die im Patiententumor tatsächlich vorliegenden molekularen Zielstrukturen. Zusätzlich wird die Medikamentenauswahl und -dosierung an die individuelle Stoffwechselleistung des einzelnen Patienten optimal angepasst [13]. Durch die individualisierte Therapie können den Patienten wirksamere und sicherere Therapieoptionen angeboten werden. Diese Entwicklung in der Therapie zeichnet sich klar ab: immer stärker zielen Strategien darauf ab, neue Medikamente nur bei jenen Patienten anzuwenden, die voraussichtlich am meisten davon profitieren und die wenigsten Nebenwirkungen haben werden. Die Ära der personalisierten Therapie in der Onkologie eingeläutet haben die Medikamente Trastuzumab Ende der 90er Jahre für Frauen mit HER2-positivem Brustkrebs [31] und der Tyrosinkinase-Inhibitor Imatinib zur Behandlung von Patienten mit chronischer myeloischer Leukämie (CML) und BCR-ABL Translokation im Jahr 2001 [8]. Zahlreiche neue Zielmoleküle wurden auch bereits für pädiatrische Tumorentitäten identifiziert, in Zellkulturmodellen charakterisiert und in transgenen Mausmodellen validiert. So kann schrittweise die biologische Bedeutung zahlreicher viel versprechender Moleküle für die Krebszelle verstanden werden. Dieses Verständnis ist die Grundlage für die Entwicklung innovativer, zielgerichteter Medikamente. Herausragende aktuelle Beispiele für erfolgreiche pädiatrisch-onkologische Forschung in diesem Gebiet sind Erkenntnisse aus neuen transgenen Mausmodellen, die von Prof. Dr. med. Johannes Schulte, Universitätsklinikum Essen, entwickelt wurden und für die er den diesjährigen Kind-Philipp-Preis erhält [15,22]. Auch hierzu herzlichste Glückwünsche! Ein umfassender Review von Frau Prof. Dr. med. Simone Fulda in dieser Ausgabe der Klinischen Pädiatrie (S. 115-119) beleuchtet die Erkenntnisse zu neuen zielgerichteten Therapiemöglichkeiten bei Rhabdomyosarkomen mit einem Fokus auf Sonic-Hedgehog and Apoptose-Signalwege. Zahlreiche weitere Aspekte personalisierter Diagnostik und molekular zielgerichteter Therapien sind in den Abstracts der diesjährigen Kind-Philipp Tagung (5.-8. Juni 2013, Wilsede, Lüneburger Heide) dargestellt.

\section{Bedarf der personalisierten Medizin an Biobanken und Datenbanken $\nabla$}

Die personalisierte Medizin benötigt neben qualitätsgesicherten Biomaterialbanken kombiniert mit zuverlässigen klinischen Daten auch neue Datenbanklösungen für molekulare Datensätze. Im EU-Projekt „p-medicine“ bauen derzeit 20 Institutionen aus 11 Ländern eine hochkomplexe IT-Infrastruktur für eine personalisierte Medizin auf. Dabei sollen patientenbezogene klinische Daten, bildgebende Daten aus der Radiologie, Labor- und molekulargenetische Daten eines Tumorpatienten IT-gestützt so zusammengeführt werden, dass unter Berücksichtigung aller Aspekte des Datenschutzes und der Datensicherheit personenbezogene Risikofaktoren ermittelt werden können. Ziel ist es außerdem, die Krankheitsdaten eines Patienten mit den molekulargenetischen Daten seines Tumors und weiterer Forschungsdaten zu dem speziellen Tumor direkt zu verzahnen und auszuwerten.

\section{Ausblick \\ $\nabla$}

Es ist absehbar, dass molekulare Hochdurchsatzverfahren zur Analyse des Genoms, Transkriptoms und Epigenoms in naher Zukunft bereits in der Routinediagnostik krebskranker Kinder eingesetzt werden können. Die „Diagnose und Therapieempfehlung aus dem Computer" und die Erfahrung des Arztes werden sich dabei im Idealfall ergänzen und schrittweise auf das Ziel der personalisierten Krebsmedizin hinarbeiten, den richtigen Patienten zur richtigen Zeit mit dem richtigen Medikament zu behandeln. Vielversprechende molekular gezielte Medikamente für eine Therapie, die für jeden individuellen Tumor maßgeschneidert ist, sind in der Entwicklung oder werden bereits in frühen klinischen Studien evaluiert [4,11,12,29,31,41,45]. Auch hierbei dürfen wir allerdings den kritischen Blick auf mögliche Nebenwirkungen nicht außer Acht lassen, wie der Beitrag 
von M. Suttorp et al. (S. 120-126) in dieser Ausgabe der Klinischen Pädiatrie am Beispiel von Tyrosinkinase-Inhibitoren bei der CML zeigt.

Angelika Eggert

\section{Literatur}

1 Blackmore C, Coppes MJ, Narendran A. Candidate genes and potential targets for therapeutics in Wilms' tumour. Clin Transl Oncol 2010; 12: 597-605

2 Bochennek $K$, Esser $R$, Lehrnbecher $T$ et al. Impact of minimal residual disease detection prior to autologous stem cell transplantation for post-transplant outcome in high risk neuroblastoma. Klin Padiatr 2012; 224: 139-142

3 Broadhead ML, Clark JC, Myers DE et al. The molecular pathogenesis of osteosarcoma: a review. Sarcoma 2011; doi: 10.1155/2011/959248

4 Corbacioglu $S$. Early phase clinical trials in pediatric hematology and oncology. Klin Padiatr 2012; 224: 197-200

5 Creutzig U, Henze G, Bielack S et al. Krebserkrankungen im Kindesalter. Erfolg durch einheitliche Therapiekonzepte seit 25 Jahren. Dtsch Ärzteblatt 2003; 100: A842-A852

6 De Preter K, Mestdagh P, Vermeulen J et al. miRNA expression profiling enables risk stratification in archived and fresh neuroblastoma tumor samples. Clin Cancer Res 2011; 17: 7684-7692

7 De Preter K, Vermeulen J, Brors B et al. Accurate outcome prediction in neuroblastoma across independent data sets using a multigene signature. Clin Cancer Res 2010; 16: 1532-1541

8 Druker BJ, Talpaz M, Resta DJ et al. Efficacy and safety of a specific inhibitor of the BCR-ABL tyrosine kinase in chronic myeloid leukemia. N Engl J Med 2001; 344: 1031-1037

9 Fischer M, Bauer T, Oberthür A et al. Integrated genomic profiling identifies two distinct molecular subtypes with divergent outcome in neuroblastoma with loss of chromosome 11q. Oncogene 2010; 29: 865-875

10 Fischer M, Spitz R, Oberthür A et al. Risk estimation of neuroblastoma patients using molecular markers. Klin Padiatr 2008; 220: 137-146

11 Grunewald TG, Greulich N, Kontny $U$ et al. Targeted therapeutics in treatment of children and young adults with solid tumors: an expert survey and review of the literature. Klin Padiatr 2012; 224: 124-131

12 Gustafson WC, Matthay KK. Progress towards personalized therapeutics: biologic- and risk-directed therapy for neuroblastoma. Expert Rev Neurother 2011; 11: 1411-1423

13 Haase R, Elsner K, Merkel $N$ et al. High dose methotrexate treatment in childhood ALL: pilot study on the impact of the MTHFR 677C $>$ T and $1298 \mathrm{~A}>\mathrm{C}$ polymorphisms on MTX-related toxicity. Klin Padiatr 2012; 224: 156-159

14 Hennes E, Zahn S, Lopes LF et al. Molecular genetic analysis of bilateral ovarian germ cell tumors. Klin Padiatr 2012; 224: 359-365

15 Heukamp LC, Thor T, Schramm A et al. Targeted expression of mutated ALK induces neuroblastoma in transgenic mice. Science Translat Med 2012; 4: 141 ra91

16 Jain $S, X u R$, Prieto VG et al. Molecular classification of soft tissue sarcomas and its clinical applications. Int J Clin Exp Pathol 2010; 3: 416-428

17 Janoueix-Lerosey I, Schleiermacher G, Michels E et al. Overall genomic pattern is a predictor of outcome in neuroblastoma. J Clin Oncol 2009; 27: 1026-1033

18 Jones DT, Jäger $N$, Kool $M$ et al. Dissecting the genomic complexity underlying medulloblastoma. Nature 2012; 488: 100-105

$19 \mathrm{Kool} \mathrm{M}$, Korshunov A, Remke $M$ et al. Molecular subgroups of medulloblastoma: an international meta-analysis of transcriptome, genetic aberrations, and clinical data of WNT, SHH, Group 3, and Group 4 medulloblastomas. Acta Neuropathol 2012; 123: 473-484

20 Lawrenz B, Henes $M$, Neunhoeffer $E$ et al. Fertility preservation in girls and adolescents before chemotherapy and radiation - review of the literature. Klin Padiatr 2011; 223: 126-130

21 Maris JM. Recent advances in neuroblastoma. N Engl J Med 2010; 362: 2202-2211

22 Molenaar JJ, Domingo-Fernández R, Ebus ME et al. LIN28B induces neuroblastoma and enhances MYCN levels via let-7 suppression. Nat Genet 2012; 44: 1199-1206
23 Northcott PA, Jones DT, Kool M et al. Medulloblastomics: the end of the beginning. Nat Rev Cancer 2012; 12: 818-834

24 Northcott PA, Korshunov A, Pfister SM et al. The clinical implications of medulloblastoma subgroups. Nat Rev Neurol 2012; 8: 340-351

25 Northcott PA, Korshunov A, Witt $H$ et al. Medulloblastoma comprises four distinct molecular variants. J Clin Oncol 2011; 29: 1408-1414

26 Northcott PA, Shih DJ, Peacock J et al. Subgroup-specific structural variation across 1,000 medulloblastoma genomes. Nature 2012; 488: 49-56

27 Oberthuer A, Berthold F, Warnat P et al. Customized oligonucleotide microarray gene expression-based classification of neuroblastoma patients outperforms current clinical risk stratification. J Clin Oncol 2006; 24: 5070-5078

28 Oberthuer A, Hero B, Berthold $F$ et al. Prognostic impact of gene expression-based classification for neuroblastoma. J Clin Oncol 2010; 28: 3506-3515

29 Øra I, Eggert A. Progress in treatment and risk stratification of neuroblastoma: impact on future clinical and basic research. Semin Cancer Biol 2011; 21: 217-228

30 Park JR, Eggert A, Caron H. Neuroblastoma: biology, prognosis, and treatment. Pediatr Clin North Am. 2008; 55: 97-120

31 Pegram MD, Lipton A, Hayes DF et al. Phase II study of receptor-enhanced chemosensitivity using recombinant humanized anti-p185HER2/ neu monoclonal antibody plus cisplatin in patients with HER2/neuoverexpressing metastatic breast cancer refractory to chemotherapy treatment. J Clin Oncol 1998; 16: 2659-2671

32 Potratz J, Jürgens H, Craft A et al. Ewing sarcoma: biology-based therapeutic perspectives. Pediatr Hematol Oncol 2012; 29: 12-27

33 Pugh TJ, Weeraratne SD, Archer TC et al. Medulloblastoma exome sequencing uncovers subtype-specific somatic mutations. Nature 2012; 488: $106-110$

34 Rausch T, Jones DT, Zapatka $M$ et al. Genome sequencing of pediatric medulloblastoma links catastrophic DNA rearrangements with TP53 mutations. Cell 2012; 148: 59-71

35 Reinhardt D, Von Neuhoff C, Sander A et al. Genetic prognostic factors in childhood acute myeloid leukemia. Klin Padiatr 2012; 224: 372-376

36 Rossig C, Juergens H. Aetiology of childhood acute leukaemias: current status of knowledge. Radiat Prot Dosimetry 2008; 132: 114-118

37 Royer-Pokora B, Graf N. Wilms tumors arising at young age: a genetic basis to distinguish subgroups for individualized therapy. J Clin Oncol 2011; 29: e485-e486

38 Schramm A, Schulte JH, Klein-Hitpass L et al. Prediction of clinical outcome and biological characterization of neuroblastoma by expression profiling. Oncogene 2005; 24: 7902-7912

39 Schwartzentruber J, Korshunov A, Liu XY et al. Driver mutations in histone $\mathrm{H} 3.3$ and chromatin remodelling genes in paediatric glioblastoma. Nature 2012; 482: 226-231

40 Speleman F, De Preter K, Vandesompele J. Neuroblastoma genetics and phenotype: a tale of heterogeneity. Semin Cancer Biol 2011; 21: 238-244

41 Stanulla M, Cario G, Meissner B et al. Integrating molecular information into treatment of childhood acute lymphoblastic leukemia - a perspective from the BFM Study Group. Blood Cells Mol Dis 2007; 39: 160-163

42 Taylor MD, Northcott PA, Korshunov A et al. Molecular subgroups of medulloblastoma: the current consensus. Acta Neuropathol 2012; 123: 465-472

43 Vermeulen J, De Preter K, Naranjo A et al. Predicting outcomes for children with neuroblastoma using a multigene-expression signature: a retrospective SIOPEN/COG/GPOH study. Lancet Oncol 2009; 10: 663-671

44 Witt H, Mack SC, Ryzhova M et al. Delineation of two clinically and molecularly distinct subgroups of posterior fossa ependymoma. Cancer Cell 2011; 20: 143-157

45 Witt 0 , Milde T, Deubzer HE et al. Phase I/II intra-patient dose escalation study of vorinostat in children with relapsed solid tumor, lymphoma or leukemia. Klin Padiatr 2012; 224: 398-403

46 Wittmann S, Wunder C, Zirn B et al. New prognostic markers revealed by evaluation of genes correlated with clinical parameters in Wilms tumors. Genes Chromosomes Cancer 2008; 47: 386-395 